\title{
Drug-Specific Design of Telodendrimer Architecture for Effective Doxorubicin Encapsulation
}

Wenjuan Jiang, ${ }^{\dagger}$ Xiaoyi Wang, ${ }^{\dagger}$ Dandan Guo, ${ }^{\ddagger}$ Juntao Luo $^{*}$ and Shikha Nangia ${ }^{\dagger *}$

${ }^{*}$ Department of Pharmacology, Upstate Cancer Center, SUNY Upstate Medical University, Syracuse, NY 13210

'Department of Biomedical and Chemical Engineering, Syracuse University, Syracuse NY 13244

*To whom correspondence should be addressed.

Phone: 315-443-0571

Email: snangia@syr.edu 


\section{Supporting Information:}

Table S1. GROMACS topology for coarse grained $\mathrm{PEG}^{5 \mathrm{~K}} \mathrm{CA}_{4} \mathrm{RH}_{4}$ telodendrimer.

\begin{tabular}{clrllrr}
\hline nr & type & resnr & residue & atom & cgnr & charge \\
\hline 1 & SN0 & 1 & PEO & O1 & 1 & 0 \\
2 & SN0 & 1 & PEO & O1 & 2 & 0 \\
3 & SN0 & 1 & PEO & O1 & 3 & 0 \\
4 & SN0 & 1 & PEO & O1 & 4 & 0 \\
5 & SN0 & 1 & PEO & O1 & 5 & 0 \\
6 & SN0 & 1 & PEO & O1 & 6 & 0 \\
7 & SN0 & 1 & PEO & O1 & 7 & 0 \\
8 & SN0 & 1 & PEO & O1 & 8 & 0 \\
9 & SN0 & 1 & PEO & O1 & 9 & 0 \\
10 & SN0 & 1 & PEO & O1 & 10 & 0 \\
11 & SN0 & 1 & PEO & O1 & 11 & 0 \\
12 & SN0 & 1 & PEO & O1 & 12 & 0 \\
13 & SN0 & 1 & PEO & O1 & 13 & 0 \\
14 & SN0 & 1 & PEO & O1 & 14 & 0 \\
15 & SN0 & 1 & PEO & O1 & 15 & 0 \\
16 & SN0 & 1 & PEO & O1 & 16 & 0 \\
17 & SN0 & 1 & PEO & O1 & 17 & 0 \\
18 & SN0 & 1 & PEO & O1 & 18 & 0 \\
19 & SN0 & 1 & PEO & O1 & 19 & 0 \\
20 & SN0 & 1 & PEO & O1 & 20 & 0 \\
21 & SN0 & 1 & PEO & O1 & 21 & 0 \\
22 & SN0 & 1 & PEO & O1 & 22 & 0 \\
23 & SN0 & 1 & PEO & O1 & 23 & 0 \\
24 & SN0 & 1 & PEO & O1 & 24 & 0 \\
25 & SN0 & 1 & PEO & O1 & 25 & 0 \\
26 & SN0 & 1 & PEO & O1 & 26 & 0 \\
27 & SN0 & 1 & PEO & O1 & 27 & 0 \\
28 & SN0 & 1 & PEO & O1 & 28 & 0 \\
29 & SN0 & 1 & PEO & O1 & 29 & 0 \\
30 & SN0 & 1 & PEO & O1 & 30 & 0 \\
31 & SN0 & 1 & PEO & O1 & 31 & 0 \\
32 & SN0 & 1 & PEO & O1 & 32 & 0 \\
33 & SN0 & 1 & PEO & O1 & 33 & 0 \\
34 & SN0 & 1 & PEO & O1 & 34 & 0 \\
\hline & & & & & &
\end{tabular}




\begin{tabular}{|c|c|c|c|c|c|c|}
\hline 35 & SN0 & 1 & PEO & $\mathrm{O} 1$ & 35 & 0 \\
\hline 36 & SN0 & 1 & PEO & $\mathrm{O} 1$ & 36 & 0 \\
\hline 37 & SN0 & 1 & PEO & $\mathrm{O} 1$ & 37 & 0 \\
\hline 38 & SN0 & 1 & PEO & $\mathrm{O} 1$ & 38 & 0 \\
\hline 39 & SN0 & 1 & PEO & $\mathrm{O} 1$ & 39 & 0 \\
\hline 40 & SN0 & 1 & PEO & $\mathrm{O} 1$ & 40 & 0 \\
\hline 41 & SN0 & 1 & PEO & $\mathrm{O} 1$ & 41 & 0 \\
\hline 42 & SN0 & 1 & PEO & $\mathrm{O} 1$ & 42 & 0 \\
\hline 43 & SN0 & 1 & PEO & $\mathrm{O} 1$ & 43 & 0 \\
\hline 44 & SN0 & 1 & PEO & $\mathrm{O} 1$ & 44 & 0 \\
\hline 45 & SN0 & 1 & PEO & $\mathrm{O} 1$ & 45 & 0 \\
\hline 46 & SN0 & 1 & PEO & $\mathrm{O} 1$ & 46 & 0 \\
\hline 47 & SN0 & 1 & PEO & $\mathrm{O} 1$ & 47 & 0 \\
\hline 48 & SN0 & 1 & PEO & $\mathrm{O} 1$ & 48 & 0 \\
\hline 49 & SN0 & 1 & PEO & $\mathrm{O} 1$ & 49 & 0 \\
\hline 50 & SN0 & 1 & PEO & $\mathrm{O} 1$ & 50 & 0 \\
\hline 51 & SN0 & 1 & PEO & $\mathrm{O} 1$ & 51 & 0 \\
\hline 52 & SN0 & 1 & PEO & $\mathrm{O} 1$ & 52 & 0 \\
\hline 53 & SN0 & 1 & PEO & $\mathrm{O} 1$ & 53 & 0 \\
\hline 54 & SN0 & 1 & PEO & $\mathrm{O} 1$ & 54 & 0 \\
\hline 55 & SN0 & 1 & PEO & $\mathrm{O} 1$ & 55 & 0 \\
\hline 56 & SN0 & 1 & PEO & $\mathrm{O} 1$ & 56 & 0 \\
\hline 57 & SN0 & 1 & PEO & $\mathrm{O} 1$ & 57 & 0 \\
\hline 58 & SN0 & 1 & PEO & $\mathrm{O} 1$ & 58 & 0 \\
\hline 59 & SN0 & 1 & PEO & $\mathrm{O} 1$ & 59 & 0 \\
\hline 60 & SN0 & 1 & PEO & $\mathrm{O} 1$ & 60 & 0 \\
\hline 61 & SN0 & 1 & PEO & $\mathrm{O} 1$ & 61 & 0 \\
\hline 62 & SN0 & 1 & PEO & $\mathrm{O} 1$ & 62 & 0 \\
\hline 63 & SN0 & 1 & PEO & $\mathrm{O} 1$ & 63 & 0 \\
\hline 64 & SN0 & 1 & PEO & $\mathrm{O} 1$ & 64 & 0 \\
\hline 65 & SN0 & 1 & PEO & $\mathrm{O} 1$ & 65 & 0 \\
\hline 66 & SN0 & 1 & PEO & $\mathrm{O} 1$ & 66 & 0 \\
\hline 67 & SN0 & 1 & PEO & $\mathrm{O} 1$ & 67 & 0 \\
\hline 68 & SN0 & 1 & PEO & $\mathrm{O} 1$ & 68 & 0 \\
\hline 69 & SN0 & 1 & PEO & $\mathrm{O} 1$ & 69 & 0 \\
\hline 70 & SN0 & 1 & PEO & $\mathrm{O} 1$ & 70 & 0 \\
\hline 71 & SN0 & 1 & PEO & $\mathrm{O} 1$ & 71 & 0 \\
\hline 72 & SN0 & 1 & PEO & $\mathrm{O} 1$ & 72 & 0 \\
\hline 73 & SN0 & 1 & PEO & $\mathrm{O} 1$ & 73 & 0 \\
\hline 74 & SN0 & 1 & PEO & $\mathrm{O} 1$ & 74 & 0 \\
\hline 75 & SN0 & 1 & PEO & $\mathrm{O} 1$ & 75 & 0 \\
\hline 76 & SN0 & 1 & PEO & $\mathrm{O} 1$ & 76 & 0 \\
\hline
\end{tabular}




\begin{tabular}{|c|c|c|c|c|c|c|}
\hline 77 & SN0 & 1 & PEO & O1 & 77 & 0 \\
\hline 78 & SN0 & 1 & PEO & $\mathrm{O} 1$ & 78 & 0 \\
\hline 79 & SN0 & 1 & PEO & O1 & 79 & 0 \\
\hline 80 & SN0 & 1 & PEO & $\mathrm{O} 1$ & 80 & 0 \\
\hline 81 & SN0 & 1 & PEO & $\mathrm{O} 1$ & 81 & 0 \\
\hline 82 & SN0 & 1 & PEO & $\mathrm{O} 1$ & 82 & 0 \\
\hline 83 & SN0 & 1 & PEO & O1 & 83 & 0 \\
\hline 84 & SN0 & 1 & PEO & O1 & 84 & 0 \\
\hline 85 & SN0 & 1 & PEO & O1 & 85 & 0 \\
\hline 86 & SN0 & 1 & PEO & $\mathrm{O} 1$ & 86 & 0 \\
\hline 87 & SN0 & 1 & PEO & O1 & 87 & 0 \\
\hline 88 & SN0 & 1 & PEO & O1 & 88 & 0 \\
\hline 89 & SN0 & 1 & PEO & $\mathrm{O} 1$ & 89 & 0 \\
\hline 90 & SN0 & 1 & PEO & $\mathrm{O} 1$ & 90 & 0 \\
\hline 91 & SN0 & 1 & PEO & O1 & 91 & 0 \\
\hline 92 & SN0 & 1 & PEO & O1 & 92 & 0 \\
\hline 93 & SN0 & 1 & PEO & $\mathrm{O} 1$ & 93 & 0 \\
\hline 94 & SN0 & 1 & PEO & O1 & 94 & 0 \\
\hline 95 & SN0 & 1 & PEO & O1 & 95 & 0 \\
\hline 96 & SN0 & 1 & PEO & O1 & 96 & 0 \\
\hline 97 & SN0 & 1 & PEO & $\mathrm{O} 1$ & 97 & 0 \\
\hline 98 & SN0 & 1 & PEO & O1 & 98 & 0 \\
\hline 99 & SN0 & 1 & PEO & O1 & 99 & 0 \\
\hline 100 & SN0 & 1 & PEO & $\mathrm{O} 1$ & 100 & 0 \\
\hline 101 & SN0 & 1 & PEO & $\mathrm{O} 1$ & 101 & 0 \\
\hline 102 & SN0 & 1 & PEO & O1 & 102 & 0 \\
\hline 103 & SN0 & 1 & PEO & O1 & 103 & 0 \\
\hline 104 & SN0 & 1 & PEO & $\mathrm{O} 1$ & 104 & 0 \\
\hline 105 & SN0 & 1 & PEO & $\mathrm{O} 1$ & 105 & 0 \\
\hline 106 & SN0 & 1 & PEO & O1 & 106 & 0 \\
\hline 107 & SN0 & 1 & PEO & O1 & 107 & 0 \\
\hline 108 & SN0 & 1 & PEO & $\mathrm{O} 1$ & 108 & 0 \\
\hline 109 & SN0 & 1 & PEO & $\mathrm{O} 1$ & 109 & 0 \\
\hline 110 & SN0 & 1 & PEO & $\mathrm{O} 1$ & 110 & 0 \\
\hline 111 & SN0 & 1 & PEO & O1 & 111 & 0 \\
\hline 112 & SN0 & 1 & PEO & $\mathrm{O} 1$ & 112 & 0 \\
\hline 113 & P5 & 2 & LYS & $\mathrm{BB}$ & 113 & 0 \\
\hline 114 & C3 & 2 & LYS & $\mathrm{SC} 1$ & 114 & 0 \\
\hline 115 & $\mathrm{P} 1$ & 2 & LYS & $\mathrm{SC} 2$ & 115 & 0 \\
\hline 116 & P5 & 3 & LYS & BB & 116 & 0 \\
\hline 117 & C3 & 3 & LYS & $\mathrm{SC} 1$ & 117 & 0 \\
\hline 118 & $\mathrm{P} 1$ & 3 & LYS & $\mathrm{SC} 2$ & 118 & 0 \\
\hline
\end{tabular}




\begin{tabular}{|c|c|c|c|c|c|c|}
\hline 119 & P5 & 4 & LYS & BB & 119 & 0 \\
\hline 120 & C3 & 4 & LYS & SC1 & 120 & 0 \\
\hline 121 & P1 & 4 & LYS & $\mathrm{SC} 2$ & 121 & 0 \\
\hline 122 & P5 & 5 & LYS & BB & 122 & 0 \\
\hline 123 & C3 & 5 & LYS & $\mathrm{SC} 1$ & 123 & 0 \\
\hline 124 & P1 & 5 & LYS & $\mathrm{SC} 2$ & 124 & 0 \\
\hline 125 & P5 & 6 & LYS & BB & 125 & 0 \\
\hline 126 & C3 & 6 & LYS & $\mathrm{SC} 1$ & 126 & 0 \\
\hline 127 & P1 & 6 & LYS & $\mathrm{SC} 2$ & 127 & 0 \\
\hline 128 & P5 & 7 & LYS & BB & 128 & 0 \\
\hline 129 & C3 & 7 & LYS & $\mathrm{SC} 1$ & 129 & 0 \\
\hline 130 & P1 & 7 & LYS & $\mathrm{SC} 2$ & 130 & 0 \\
\hline 131 & P5 & 8 & LYS & BB & 131 & 0 \\
\hline 132 & C3 & 8 & LYS & $\mathrm{SC} 1$ & 132 & 0 \\
\hline 133 & P1 & 8 & LYS & $\mathrm{SC} 2$ & 133 & 0 \\
\hline 134 & SP5 & 9 & RHN & N1 & 134 & 0 \\
\hline 135 & SC4 & 9 & RHN & $\mathrm{N} 2$ & 135 & 0 \\
\hline 136 & $\mathrm{SC} 1$ & 9 & RHN & N3 & 136 & 0 \\
\hline 137 & P2 & 9 & RHN & N4 & 137 & 0 \\
\hline 138 & P2 & 9 & RHN & N5 & 138 & 0 \\
\hline 139 & $\mathrm{SC} 4$ & 9 & RHN & N6 & 139 & 0 \\
\hline 140 & $\mathrm{SC} 1$ & 9 & RHN & N7 & 140 & 0 \\
\hline 141 & SP5 & 9 & RHN & N8 & 141 & 0 \\
\hline 142 & P3 & 9 & RHN & N9 & 142 & 0 \\
\hline 143 & SP5 & 10 & CHOA & $\mathrm{OH}$ & 143 & 0 \\
\hline 144 & $\mathrm{SC} 1$ & 10 & CHOA & R1 & 144 & 0 \\
\hline 145 & $\mathrm{SC} 3$ & 10 & CHOA & $\mathrm{R} 2$ & 145 & 0 \\
\hline 146 & SP5 & 10 & CHOA & $\mathrm{O} 2$ & 146 & 0 \\
\hline 147 & SP5 & 10 & CHOA & $\mathrm{O} 3$ & 147 & 0 \\
\hline 148 & $\mathrm{SC} 1$ & 10 & CHOA & R3 & 148 & 0 \\
\hline 149 & $\mathrm{SC} 1$ & 10 & CHOA & $\mathrm{C} 1$ & 149 & 0 \\
\hline 150 & SP5 & 11 & CHOA & $\mathrm{OH}$ & 150 & 0 \\
\hline 151 & $\mathrm{SC} 1$ & 11 & $\mathrm{CHOA}$ & $\mathrm{R} 1$ & 151 & 0 \\
\hline 152 & $\mathrm{SC} 3$ & 11 & CHOA & $\mathrm{R} 2$ & 152 & 0 \\
\hline 153 & SP5 & 11 & CHOA & $\mathrm{O} 2$ & 153 & 0 \\
\hline 154 & SP5 & 11 & CHOA & $\mathrm{O} 3$ & 154 & 0 \\
\hline 155 & $\mathrm{SC} 1$ & 11 & CHOA & R3 & 155 & 0 \\
\hline 156 & $\mathrm{SC} 1$ & 11 & CHOA & $\mathrm{C} 1$ & 156 & 0 \\
\hline 157 & SP5 & 12 & RHN & N1 & 157 & 0 \\
\hline 158 & SC4 & 12 & RHN & $\mathrm{N} 2$ & 158 & 0 \\
\hline 159 & $\mathrm{SC} 1$ & 12 & RHN & N3 & 159 & 0 \\
\hline 160 & P2 & 12 & RHN & N4 & 160 & 0 \\
\hline
\end{tabular}




\begin{tabular}{lllllll}
\hline 161 & P2 & 12 & RHN & N5 & 161 & 0 \\
162 & SC4 & 12 & RHN & N6 & 162 & 0 \\
163 & SC1 & 12 & RHN & N7 & 163 & 0 \\
164 & SP5 & 12 & RHN & N8 & 164 & 0 \\
165 & P3 & 12 & RHN & N9 & 165 & 0 \\
166 & SP5 & 13 & CHOA & OH & 166 & 0 \\
167 & SC1 & 13 & CHOA & R1 & 167 & 0 \\
168 & SC3 & 13 & CHOA & R2 & 168 & 0 \\
169 & SP5 & 13 & CHOA & O2 & 169 & 0 \\
170 & SP5 & 13 & CHOA & O3 & 170 & 0 \\
171 & SC1 & 13 & CHOA & R3 & 171 & 0 \\
172 & SC1 & 13 & CHOA & C1 & 172 & 0 \\
173 & SP5 & 14 & RHN & N1 & 173 & 0 \\
174 & SC4 & 14 & RHN & N2 & 174 & 0 \\
175 & SC1 & 14 & RHN & N3 & 175 & 0 \\
176 & P2 & 14 & RHN & N4 & 176 & 0 \\
177 & P2 & 14 & RHN & N5 & 177 & 0 \\
178 & SC4 & 14 & RHN & N6 & 178 & 0 \\
179 & SC1 & 14 & RHN & N7 & 179 & 0 \\
180 & SP5 & 14 & RHN & N8 & 180 & 0 \\
181 & P3 & 14 & RHN & N9 & 181 & 0 \\
182 & SP5 & 15 & CHOA & OH & 182 & 0 \\
183 & SC1 & 15 & CHOA & R1 & 183 & 0 \\
184 & SC3 & 15 & CHOA & R2 & 184 & 0 \\
185 & SP5 & 15 & CHOA & O2 & 185 & 0 \\
186 & SP5 & 15 & CHOA & O3 & 186 & 0 \\
187 & SC1 & 15 & CHOA & R3 & 187 & 0 \\
188 & SC1 & 15 & CHOA & C1 & 188 & 0 \\
189 & SP5 & 16 & RHN & N1 & 189 & 0 \\
190 & SC4 & 16 & RHN & N2 & 190 & 0 \\
191 & SC1 & 16 & RHN & N3 & 191 & 0 \\
192 & P2 & 16 & RHN & N4 & 192 & 0 \\
193 & P2 & 16 & RHN & N5 & 193 & 0 \\
194 & SC4 & 16 & RHN & N6 & 194 & 0 \\
195 & SC1 & 16 & RHN & N7 & 195 & 0 \\
196 & SP5 & 16 & RHN & N8 & 196 & 0 \\
197 & P3 & 167 & 0 \\
\hline & & & & &
\end{tabular}


Table S2. Bond length parameters for coarse-grained $\mathrm{PEG}^{5 \mathrm{~K}} \mathrm{CA}_{4} \mathrm{RH}_{4}$ telodendrimer.

\begin{tabular}{ccrrr}
\hline $\mathrm{i}$ & $\mathrm{j}$ & funct & length & force.c. \\
\hline 1 & 2 & 1 & 0.33 & 17000 \\
2 & 3 & 1 & 0.33 & 17000 \\
3 & 4 & 1 & 0.33 & 17000 \\
4 & 5 & 1 & 0.33 & 17000 \\
5 & 6 & 1 & 0.33 & 17000 \\
6 & 7 & 1 & 0.33 & 17000 \\
7 & 8 & 1 & 0.33 & 17000 \\
8 & 9 & 1 & 0.33 & 17000 \\
9 & 10 & 1 & 0.33 & 17000 \\
10 & 11 & 1 & 0.33 & 17000 \\
11 & 12 & 1 & 0.33 & 17000 \\
12 & 13 & 1 & 0.33 & 17000 \\
13 & 14 & 1 & 0.33 & 17000 \\
14 & 15 & 1 & 0.33 & 17000 \\
15 & 16 & 1 & 0.33 & 17000 \\
16 & 17 & 1 & 0.33 & 17000 \\
17 & 18 & 1 & 0.33 & 17000 \\
18 & 19 & 1 & 0.33 & 17000 \\
19 & 20 & 1 & 0.33 & 17000 \\
20 & 21 & 1 & 0.33 & 17000 \\
21 & 22 & 1 & 0.33 & 17000 \\
22 & 23 & 1 & 0.33 & 17000 \\
23 & 24 & 1 & 0.33 & 17000 \\
24 & 25 & 1 & 0.33 & 17000 \\
25 & 26 & 1 & 0.33 & 17000 \\
26 & 27 & 1 & 0.33 & 17000 \\
27 & 28 & 1 & 0.33 & 17000 \\
28 & 29 & 1 & 0.33 & 17000 \\
29 & 30 & 1 & 0.33 & 17000 \\
30 & 31 & 1 & 0.33 & 17000 \\
31 & 32 & 1 & 0.33 & 17000 \\
32 & 33 & 1 & 0.33 & 17000 \\
33 & 34 & 1 & 0.33 & 17000 \\
34 & 35 & 1 & 0.33 & 17000 \\
35 & 36 & 1 & 0.33 & 17000 \\
36 & 37 & 1 & 0.33 & 17000 \\
37 & 38 & 1 & 0.33 & 17000 \\
38 & 39 & 1 & 0.33 & 17000 \\
39 & 40 & 1 & 0.33 & 17000 \\
40 & 41 & 1 & 0.33 & 17000 \\
\hline & & & & \\
& & &
\end{tabular}




\begin{tabular}{lllll}
\hline 41 & 42 & 1 & 0.33 & 17000 \\
42 & 43 & 1 & 0.33 & 17000 \\
43 & 44 & 1 & 0.33 & 17000 \\
44 & 45 & 1 & 0.33 & 17000 \\
45 & 46 & 1 & 0.33 & 17000 \\
46 & 47 & 1 & 0.33 & 17000 \\
47 & 48 & 1 & 0.33 & 17000 \\
48 & 49 & 1 & 0.33 & 17000 \\
49 & 50 & 1 & 0.33 & 17000 \\
50 & 51 & 1 & 0.33 & 17000 \\
51 & 52 & 1 & 0.33 & 17000 \\
52 & 53 & 1 & 0.33 & 17000 \\
53 & 54 & 1 & 0.33 & 17000 \\
54 & 55 & 1 & 0.33 & 17000 \\
55 & 56 & 1 & 0.33 & 17000 \\
56 & 57 & 1 & 0.33 & 17000 \\
57 & 58 & 1 & 0.33 & 17000 \\
58 & 59 & 1 & 0.33 & 17000 \\
59 & 60 & 1 & 0.33 & 17000 \\
60 & 61 & 1 & 0.33 & 17000 \\
61 & 62 & 1 & 0.33 & 17000 \\
62 & 63 & 1 & 0.33 & 17000 \\
63 & 64 & 1 & 0.33 & 17000 \\
64 & 65 & 1 & 0.33 & 17000 \\
65 & 66 & 1 & 0.33 & 17000 \\
66 & 67 & 1 & 0.33 & 17000 \\
67 & 68 & 1 & 0.33 & 17000 \\
68 & 69 & 1 & 0.33 & 17000 \\
69 & 70 & 1 & 0.33 & 17000 \\
70 & 71 & 1 & 0.33 & 17000 \\
71 & 72 & 1 & 0.33 & 17000 \\
72 & 73 & 1 & 0.33 & 17000 \\
73 & 74 & 1 & 0.33 & 17000 \\
74 & 75 & 1 & 0.33 & 17000 \\
75 & 76 & 1 & 0.33 & 17000 \\
76 & 77 & 1 & 0.33 & 17000 \\
77 & 78 & 1 & 0.33 & 17000 \\
78 & 79 & 1 & 0.33 & 17000 \\
79 & 80 & 1 & 0.33 & 17000 \\
80 & 81 & 1 & 0.33 & 17000 \\
81 & 82 & 1 & 0.33 & 17000 \\
82 & 83 & 1 & 0.33 & 17000 \\
\hline & & & & \\
\hline & & &
\end{tabular}




\begin{tabular}{|c|c|c|c|c|}
\hline 83 & 84 & 1 & 0.33 & 17000 \\
\hline 84 & 85 & 1 & 0.33 & 17000 \\
\hline 85 & 86 & 1 & 0.33 & 17000 \\
\hline 86 & 87 & 1 & 0.33 & 17000 \\
\hline 87 & 88 & 1 & 0.33 & 17000 \\
\hline 88 & 89 & 1 & 0.33 & 17000 \\
\hline 89 & 90 & 1 & 0.33 & 17000 \\
\hline 90 & 91 & 1 & 0.33 & 17000 \\
\hline 91 & 92 & 1 & 0.33 & 17000 \\
\hline 92 & 93 & 1 & 0.33 & 17000 \\
\hline 93 & 94 & 1 & 0.33 & 17000 \\
\hline 94 & 95 & 1 & 0.33 & 17000 \\
\hline 95 & 96 & 1 & 0.33 & 17000 \\
\hline 96 & 97 & 1 & 0.33 & 17000 \\
\hline 97 & 98 & 1 & 0.33 & 17000 \\
\hline 98 & 99 & 1 & 0.33 & 17000 \\
\hline 99 & 100 & 1 & 0.33 & 17000 \\
\hline 100 & 101 & 1 & 0.33 & 17000 \\
\hline 101 & 102 & 1 & 0.33 & 17000 \\
\hline 102 & 103 & 1 & 0.33 & 17000 \\
\hline 103 & 104 & 1 & 0.33 & 17000 \\
\hline 104 & 105 & 1 & 0.33 & 17000 \\
\hline 105 & 106 & 1 & 0.33 & 17000 \\
\hline 106 & 107 & 1 & 0.33 & 17000 \\
\hline 107 & 108 & 1 & 0.33 & 17000 \\
\hline 108 & 109 & 1 & 0.33 & 17000 \\
\hline 109 & 110 & 1 & 0.33 & 17000 \\
\hline 110 & 111 & 1 & 0.33 & 17000 \\
\hline 111 & 112 & 1 & 0.33 & 17000 \\
\hline 112 & 119 & 1 & 0.33 & 1200 \\
\hline 113 & 116 & 1 & 0.35 & 1250 \\
\hline 116 & 119 & 1 & 0.35 & 1250 \\
\hline 125 & 128 & 1 & 0.35 & 1250 \\
\hline 118 & 122 & 1 & 0.35 & 1250 \\
\hline 121 & 128 & 1 & 0.35 & 1250 \\
\hline 130 & 131 & 1 & 0.35 & 1250 \\
\hline 113 & 114 & 1 & 0.33 & 5000 \\
\hline 116 & 117 & 1 & 0.33 & 5000 \\
\hline 119 & 120 & 1 & 0.33 & 5000 \\
\hline 122 & 123 & 1 & 0.33 & 5000 \\
\hline 125 & 126 & 1 & 0.33 & 5000 \\
\hline 128 & 129 & 1 & 0.33 & 5000 \\
\hline
\end{tabular}




\begin{tabular}{rrrrr}
\hline 131 & 132 & 1 & 0.33 & 5000 \\
114 & 115 & 1 & 0.28 & 5000 \\
117 & 118 & 1 & 0.28 & 5000 \\
120 & 121 & 1 & 0.28 & 5000 \\
123 & 124 & 1 & 0.28 & 5000 \\
126 & 127 & 1 & 0.28 & 5000 \\
129 & 130 & 1 & 0.28 & 5000 \\
132 & 133 & 1 & 0.28 & 5000 \\
115 & 142 & 1 & 0.368 & 20000 \\
113 & 149 & 1 & 0.368 & 20000 \\
125 & 156 & 1 & 0.368 & 20000 \\
127 & 165 & 1 & 0.368 & 20000 \\
122 & 172 & 1 & 0.368 & 20000 \\
124 & 181 & 1 & 0.368 & 20000 \\
131 & 188 & 1 & 0.368 & 20000 \\
133 & 197 & 1 & 0.368 & 20000 \\
134 & 135 & 1 & 0.27 & 9000 \\
135 & 136 & 1 & 0.27 & 9000 \\
136 & 134 & 1 & 0.27 & 9000 \\
139 & 140 & 1 & 0.27 & 9000 \\
140 & 141 & 1 & 0.27 & 9000 \\
141 & 139 & 1 & 0.27 & 9000 \\
135 & 137 & 1 & 0.27 & 9000 \\
135 & 138 & 1 & 0.27 & 9000 \\
137 & 139 & 1 & 0.27 & 9000 \\
138 & 139 & 1 & 0.27 & 9000 \\
137 & 138 & 1 & 0.415 & 9000 \\
140 & 142 & 1 & 0.231 & 9000 \\
143 & 144 & 1 & 0.242 & 20000 \\
144 & 145 & 1 & 0.26 & 20000 \\
144 & 146 & 1 & 0.341 & 20000 \\
146 & 148 & 1 & 0.213 & 20000 \\
146 & 149 & 1 & 0.544 & 20000 \\
147 & 148 & 1 & 0.203 & 20000 \\
148 & 149 & 1 & 0.368 & 20000 \\
150 & 151 & 1 & 0.242 & 20000 \\
151 & 152 & 1 & 0.26 & 20000 \\
151 & 153 & 1 & 0.341 & 20000 \\
153 & 155 & 1 & 0.213 & 20000 \\
153 & 156 & 1 & 0.544 & 20000 \\
154 & 155 & 1 & 0.203 & 20000 \\
155 & 156 & 1 & 0.368 & 20000 \\
\hline & & & & \\
\hline
\end{tabular}




\begin{tabular}{rrrrr}
\hline 157 & 158 & 1 & 0.27 & 9000 \\
158 & 159 & 1 & 0.27 & 9000 \\
159 & 157 & 1 & 0.27 & 9000 \\
162 & 163 & 1 & 0.27 & 9000 \\
163 & 164 & 1 & 0.27 & 9000 \\
164 & 162 & 1 & 0.27 & 9000 \\
158 & 160 & 1 & 0.27 & 9000 \\
158 & 161 & 1 & 0.27 & 9000 \\
160 & 162 & 1 & 0.27 & 9000 \\
161 & 162 & 1 & 0.27 & 9000 \\
160 & 161 & 1 & 0.415 & 9000 \\
163 & 165 & 1 & 0.231 & 9000 \\
166 & 167 & 1 & 0.242 & 20000 \\
167 & 168 & 1 & 0.26 & 20000 \\
167 & 169 & 1 & 0.341 & 20000 \\
169 & 171 & 1 & 0.213 & 20000 \\
169 & 172 & 1 & 0.544 & 20000 \\
170 & 171 & 1 & 0.203 & 20000 \\
171 & 172 & 1 & 0.368 & 20000 \\
173 & 174 & 1 & 0.27 & 9000 \\
174 & 175 & 1 & 0.27 & 9000 \\
175 & 173 & 1 & 0.27 & 9000 \\
178 & 179 & 1 & 0.27 & 9000 \\
179 & 180 & 1 & 0.27 & 9000 \\
180 & 178 & 1 & 0.27 & 9000 \\
174 & 176 & 1 & 0.27 & 9000 \\
174 & 177 & 1 & 0.27 & 9000 \\
176 & 178 & 1 & 0.27 & 9000 \\
177 & 178 & 1 & 0.27 & 9000 \\
176 & 177 & 1 & 0.415 & 9000 \\
179 & 181 & 1 & 0.231 & 9000 \\
182 & 183 & 1 & 0.242 & 20000 \\
183 & 184 & 1 & 0.26 & 20000 \\
183 & 185 & 1 & 0.341 & 20000 \\
185 & 187 & 1 & 0.213 & 20000 \\
185 & 188 & 1 & 0.544 & 20000 \\
186 & 187 & 1 & 0.203 & 20000 \\
187 & 188 & 1 & 0.368 & 20000 \\
189 & 190 & 1 & 0.27 & 9000 \\
190 & 191 & 1 & 0.27 & 9000 \\
191 & 189 & 1 & 0.27 & 9000 \\
194 & 195 & 1 & 0.27 & 9000 \\
\hline & & & & \\
\hline
\end{tabular}




\begin{tabular}{rrrrr}
\hline 195 & 196 & 1 & 0.27 & 9000 \\
196 & 194 & 1 & 0.27 & 9000 \\
190 & 192 & 1 & 0.27 & 9000 \\
190 & 193 & 1 & 0.27 & 9000 \\
192 & 194 & 1 & 0.27 & 9000 \\
193 & 194 & 1 & 0.27 & 9000 \\
195 & 197 & 1 & 0.231 & 9000 \\
\hline
\end{tabular}


Table S3. Angle parameters for coarse-grained $\mathrm{PEG}^{5 \mathrm{~K}} \mathrm{CA}_{4} \mathrm{RH}_{4}$ telodendrimer.

\begin{tabular}{|c|c|c|c|c|c|}
\hline $\mathrm{i}$ & $\mathrm{j}$ & $\mathrm{k}$ & funct & angle & force.c. \\
\hline 1 & 2 & 3 & 2 & 130 & 50 \\
\hline 2 & 3 & 4 & 2 & 130 & 50 \\
\hline 3 & 4 & 5 & 2 & 130 & 50 \\
\hline 4 & 5 & 6 & 2 & 130 & 50 \\
\hline 5 & 6 & 7 & 2 & 130 & 50 \\
\hline 6 & 7 & 8 & 2 & 130 & 50 \\
\hline 7 & 8 & 9 & 2 & 130 & 50 \\
\hline 8 & 9 & 10 & 2 & 130 & 50 \\
\hline 9 & 10 & 11 & 2 & 130 & 50 \\
\hline 10 & 11 & 12 & 2 & 130 & 50 \\
\hline 11 & 12 & 13 & 2 & 130 & 50 \\
\hline 12 & 13 & 14 & 2 & 130 & 50 \\
\hline 13 & 14 & 15 & 2 & 130 & 50 \\
\hline 14 & 15 & 16 & 2 & 130 & 50 \\
\hline 15 & 16 & 17 & 2 & 130 & 50 \\
\hline 16 & 17 & 18 & 2 & 130 & 50 \\
\hline 17 & 18 & 19 & 2 & 130 & 50 \\
\hline 18 & 19 & 20 & 2 & 130 & 50 \\
\hline 19 & 20 & 21 & 2 & 130 & 50 \\
\hline 20 & 21 & 22 & 2 & 130 & 50 \\
\hline 21 & 22 & 23 & 2 & 130 & 50 \\
\hline 22 & 23 & 24 & 2 & 130 & 50 \\
\hline 23 & 24 & 25 & 2 & 130 & 50 \\
\hline 24 & 25 & 26 & 2 & 130 & 50 \\
\hline 25 & 26 & 27 & 2 & 130 & 50 \\
\hline 26 & 27 & 28 & 2 & 130 & 50 \\
\hline 27 & 28 & 29 & 2 & 130 & 50 \\
\hline 28 & 29 & 30 & 2 & 130 & 50 \\
\hline 29 & 30 & 31 & 2 & 130 & 50 \\
\hline 30 & 31 & 32 & 2 & 130 & 50 \\
\hline 31 & 32 & 33 & 2 & 130 & 50 \\
\hline 32 & 33 & 34 & 2 & 130 & 50 \\
\hline 33 & 34 & 35 & 2 & 130 & 50 \\
\hline 34 & 35 & 36 & 2 & 130 & 50 \\
\hline 35 & 36 & 37 & 2 & 130 & 50 \\
\hline 36 & 37 & 38 & 2 & 130 & 50 \\
\hline 37 & 38 & 39 & 2 & 130 & 50 \\
\hline 38 & 39 & 40 & 2 & 130 & 50 \\
\hline 39 & 40 & 41 & 2 & 130 & 50 \\
\hline 40 & 41 & 42 & 2 & 130 & 50 \\
\hline
\end{tabular}




\begin{tabular}{|c|c|c|c|c|c|}
\hline 41 & 42 & 43 & 2 & 130 & 50 \\
\hline 42 & 43 & 44 & 2 & 130 & 50 \\
\hline 43 & 44 & 45 & 2 & 130 & 50 \\
\hline 44 & 45 & 46 & 2 & 130 & 50 \\
\hline 45 & 46 & 47 & 2 & 130 & 50 \\
\hline 46 & 47 & 48 & 2 & 130 & 50 \\
\hline 47 & 48 & 49 & 2 & 130 & 50 \\
\hline 48 & 49 & 50 & 2 & 130 & 50 \\
\hline 49 & 50 & 51 & 2 & 130 & 50 \\
\hline 50 & 51 & 52 & 2 & 130 & 50 \\
\hline 51 & 52 & 53 & 2 & 130 & 50 \\
\hline 52 & 53 & 54 & 2 & 130 & 50 \\
\hline 53 & 54 & 55 & 2 & 130 & 50 \\
\hline 54 & 55 & 56 & 2 & 130 & 50 \\
\hline 55 & 56 & 57 & 2 & 130 & 50 \\
\hline 56 & 57 & 58 & 2 & 130 & 50 \\
\hline 57 & 58 & 59 & 2 & 130 & 50 \\
\hline 58 & 59 & 60 & 2 & 130 & 50 \\
\hline 59 & 60 & 61 & 2 & 130 & 50 \\
\hline 60 & 61 & 62 & 2 & 130 & 50 \\
\hline 61 & 62 & 63 & 2 & 130 & 50 \\
\hline 62 & 63 & 64 & 2 & 130 & 50 \\
\hline 63 & 64 & 65 & 2 & 130 & 50 \\
\hline 64 & 65 & 66 & 2 & 130 & 50 \\
\hline 65 & 66 & 67 & 2 & 130 & 50 \\
\hline 66 & 67 & 68 & 2 & 130 & 50 \\
\hline 67 & 68 & 69 & 2 & 130 & 50 \\
\hline 68 & 69 & 70 & 2 & 130 & 50 \\
\hline 69 & 70 & 71 & 2 & 130 & 50 \\
\hline 70 & 71 & 72 & 2 & 130 & 50 \\
\hline 71 & 72 & 73 & 2 & 130 & 50 \\
\hline 72 & 73 & 74 & 2 & 130 & 50 \\
\hline 73 & 74 & 75 & 2 & 130 & 50 \\
\hline 74 & 75 & 76 & 2 & 130 & 50 \\
\hline 75 & 76 & 77 & 2 & 130 & 50 \\
\hline 76 & 77 & 78 & 2 & 130 & 50 \\
\hline 77 & 78 & 79 & 2 & 130 & 50 \\
\hline 78 & 79 & 80 & 2 & 130 & 50 \\
\hline 79 & 80 & 81 & 2 & 130 & 50 \\
\hline 80 & 81 & 82 & 2 & 130 & 50 \\
\hline 81 & 82 & 83 & 2 & 130 & 50 \\
\hline 82 & 83 & 84 & 2 & 130 & 50 \\
\hline
\end{tabular}




\begin{tabular}{|c|c|c|c|c|c|}
\hline 83 & 84 & 85 & 2 & 130 & 50 \\
\hline 84 & 85 & 86 & 2 & 130 & 50 \\
\hline 85 & 86 & 87 & 2 & 130 & 50 \\
\hline 86 & 87 & 88 & 2 & 130 & 50 \\
\hline 87 & 88 & 89 & 2 & 130 & 50 \\
\hline 88 & 89 & 90 & 2 & 130 & 50 \\
\hline 89 & 90 & 91 & 2 & 130 & 50 \\
\hline 90 & 91 & 92 & 2 & 130 & 50 \\
\hline 91 & 92 & 93 & 2 & 130 & 50 \\
\hline 92 & 93 & 94 & 2 & 130 & 50 \\
\hline 93 & 94 & 95 & 2 & 130 & 50 \\
\hline 94 & 95 & 96 & 2 & 130 & 50 \\
\hline 95 & 96 & 97 & 2 & 130 & 50 \\
\hline 96 & 97 & 98 & 2 & 130 & 50 \\
\hline 97 & 98 & 99 & 2 & 130 & 50 \\
\hline 98 & 99 & 100 & 2 & 130 & 50 \\
\hline 99 & 100 & 101 & 2 & 130 & 50 \\
\hline 100 & 101 & 102 & 2 & 130 & 50 \\
\hline 101 & 102 & 103 & 2 & 130 & 50 \\
\hline 102 & 103 & 104 & 2 & 130 & 50 \\
\hline 103 & 104 & 105 & 2 & 130 & 50 \\
\hline 104 & 105 & 106 & 2 & 130 & 50 \\
\hline 105 & 106 & 107 & 2 & 130 & 50 \\
\hline 106 & 107 & 108 & 2 & 130 & 50 \\
\hline 107 & 108 & 109 & 2 & 130 & 50 \\
\hline 108 & 109 & 110 & 2 & 130 & 50 \\
\hline 109 & 110 & 111 & 2 & 130 & 50 \\
\hline 110 & 111 & 112 & 2 & 130 & 50 \\
\hline 111 & 112 & 119 & 2 & 130 & 50 \\
\hline 112 & 119 & 120 & 2 & 130 & 50 \\
\hline 113 & 116 & 119 & 2 & 134 & 25 \\
\hline 118 & 122 & 123 & 2 & 100 & 25 \\
\hline 130 & 131 & 132 & 2 & 100 & 25 \\
\hline 116 & 113 & 114 & 2 & 100 & 25 \\
\hline 113 & 116 & 117 & 2 & 100 & 25 \\
\hline 116 & 119 & 120 & 2 & 100 & 25 \\
\hline 125 & 128 & 129 & 2 & 100 & 25 \\
\hline 113 & 114 & 115 & 2 & 180 & 25 \\
\hline 116 & 117 & 118 & 2 & 180 & 25 \\
\hline 119 & 120 & 121 & 2 & 180 & 25 \\
\hline 122 & 123 & 124 & 2 & 180 & 25 \\
\hline 125 & 126 & 127 & 2 & 180 & 25 \\
\hline
\end{tabular}




\begin{tabular}{|c|c|c|c|c|c|}
\hline 128 & 129 & 130 & 2 & 180 & 25 \\
\hline 115 & 142 & 140 & 2 & 130 & 50 \\
\hline 113 & 149 & 148 & 2 & 130 & 50 \\
\hline 125 & 156 & 155 & 2 & 130 & 50 \\
\hline 127 & 165 & 163 & 2 & 130 & 50 \\
\hline 122 & 172 & 171 & 2 & 130 & 50 \\
\hline 124 & 181 & 179 & 2 & 130 & 50 \\
\hline 131 & 188 & 187 & 2 & 130 & 50 \\
\hline 133 & 197 & 195 & 2 & 130 & 50 \\
\hline 134 & 135 & 137 & 1 & 78 & 80 \\
\hline 134 & 135 & 138 & 1 & 165 & 80 \\
\hline 134 & 135 & 136 & 1 & 76 & 80 \\
\hline 136 & 135 & 137 & 1 & 178 & 80 \\
\hline 136 & 135 & 138 & 1 & 89 & 80 \\
\hline 141 & 139 & 137 & 1 & 76 & 80 \\
\hline 141 & 139 & 138 & 1 & 165 & 80 \\
\hline 141 & 139 & 140 & 1 & 75 & 80 \\
\hline 140 & 139 & 137 & 1 & 178 & 80 \\
\hline 140 & 139 & 138 & 1 & 91 & 80 \\
\hline 139 & 140 & 142 & 1 & 178 & 80 \\
\hline 135 & 134 & 136 & 1 & 51 & 80 \\
\hline 134 & 136 & 135 & 1 & 56 & 80 \\
\hline 141 & 140 & 139 & 1 & 55 & 80 \\
\hline 139 & 141 & 140 & 1 & 48 & 80 \\
\hline 135 & 137 & 139 & 1 & 60 & 80 \\
\hline 135 & 138 & 139 & 1 & 60 & 80 \\
\hline 157 & 158 & 160 & 1 & 78 & 80 \\
\hline 157 & 158 & 161 & 1 & 165 & 80 \\
\hline 157 & 158 & 159 & 1 & 76 & 80 \\
\hline 159 & 158 & 160 & 1 & 178 & 80 \\
\hline 159 & 158 & 161 & 1 & 89 & 80 \\
\hline 164 & 162 & 160 & 1 & 76 & 80 \\
\hline 164 & 162 & 161 & 1 & 165 & 80 \\
\hline 164 & 162 & 163 & 1 & 75 & 80 \\
\hline 163 & 162 & 160 & 1 & 178 & 80 \\
\hline 163 & 162 & 161 & 1 & 91 & 80 \\
\hline 162 & 163 & 165 & 1 & 178 & 80 \\
\hline 158 & 157 & 159 & 1 & 51 & 80 \\
\hline 157 & 159 & 158 & 1 & 56 & 80 \\
\hline 164 & 163 & 162 & 1 & 55 & 80 \\
\hline 162 & 164 & 163 & 1 & 48 & 80 \\
\hline 158 & 160 & 162 & 1 & 60 & 80 \\
\hline
\end{tabular}




\begin{tabular}{|c|c|c|c|c|c|}
\hline 158 & 161 & 162 & 1 & 60 & 80 \\
\hline 173 & 174 & 176 & 1 & 78 & 80 \\
\hline 173 & 174 & 177 & 1 & 165 & 80 \\
\hline 173 & 174 & 175 & 1 & 76 & 80 \\
\hline 175 & 174 & 176 & 1 & 178 & 80 \\
\hline 175 & 174 & 177 & 1 & 89 & 80 \\
\hline 180 & 178 & 176 & 1 & 76 & 80 \\
\hline 180 & 178 & 177 & 1 & 165 & 80 \\
\hline 180 & 178 & 179 & 1 & 75 & 80 \\
\hline 179 & 178 & 176 & 1 & 178 & 80 \\
\hline 179 & 178 & 177 & 1 & 91 & 80 \\
\hline 178 & 179 & 181 & 1 & 178 & 80 \\
\hline 174 & 173 & 175 & 1 & 51 & 80 \\
\hline 173 & 175 & 174 & 1 & 56 & 80 \\
\hline 180 & 179 & 178 & 1 & 55 & 80 \\
\hline 178 & 180 & 179 & 1 & 48 & 80 \\
\hline 174 & 176 & 178 & 1 & 60 & 80 \\
\hline 174 & 177 & 178 & 1 & 60 & 80 \\
\hline 189 & 190 & 192 & 1 & 78 & 80 \\
\hline 189 & 190 & 193 & 1 & 165 & 80 \\
\hline 189 & 190 & 191 & 1 & 76 & 80 \\
\hline 191 & 190 & 192 & 1 & 178 & 80 \\
\hline 191 & 190 & 193 & 1 & 89 & 80 \\
\hline 196 & 194 & 192 & 1 & 76 & 80 \\
\hline 196 & 194 & 193 & 1 & 165 & 80 \\
\hline 196 & 194 & 195 & 1 & 75 & 80 \\
\hline 195 & 194 & 192 & 1 & 178 & 80 \\
\hline 195 & 194 & 193 & 1 & 91 & 80 \\
\hline 194 & 195 & 197 & 1 & 178 & 80 \\
\hline 190 & 189 & 191 & 1 & 51 & 80 \\
\hline 189 & 191 & 190 & 1 & 56 & 80 \\
\hline 196 & 195 & 194 & 1 & 55 & 80 \\
\hline 194 & 196 & 195 & 1 & 48 & 80 \\
\hline 190 & 192 & 194 & 1 & 60 & 80 \\
\hline 190 & 193 & 194 & 1 & 60 & 80 \\
\hline
\end{tabular}


Table S4. Constraints for coarse-grained $\mathrm{PEG}^{5 \mathrm{~K}} \mathrm{CA}_{4} \mathrm{RH}_{4}$ telodendrimer.

\begin{tabular}{ccrr}
\hline $\mathrm{i}$ & $\mathrm{j}$ & funct & length \\
143 & 145 & 1 & 0.493 \\
143 & 146 & 1 & 0.604 \\
145 & 146 & 1 & 0.272 \\
145 & 147 & 1 & 0.346 \\
146 & 147 & 1 & 0.294 \\
147 & 149 & 1 & 0.406 \\
150 & 152 & 1 & 0.493 \\
150 & 153 & 1 & 0.604 \\
152 & 153 & 1 & 0.272 \\
152 & 154 & 1 & 0.346 \\
153 & 154 & 1 & 0.294 \\
154 & 156 & 1 & 0.406 \\
166 & 168 & 1 & 0.493 \\
166 & 169 & 1 & 0.604 \\
168 & 169 & 1 & 0.272 \\
168 & 170 & 1 & 0.346 \\
169 & 170 & 1 & 0.294 \\
170 & 172 & 1 & 0.406 \\
182 & 184 & 1 & 0.493 \\
182 & 185 & 1 & 0.604 \\
184 & 185 & 1 & 0.272 \\
184 & 186 & 1 & 0.346 \\
185 & 186 & 1 & 0.294 \\
186 & 188 & 1 & 0.406 \\
\hline
\end{tabular}


Table S5. GROMACS topology for coarse grained $\mathrm{PEG}^{5 \mathrm{~K}} \mathrm{CA}_{4}-\mathrm{L}_{-}-\mathrm{RH}_{4}$ telodendrimer.

\begin{tabular}{|c|c|c|c|c|c|c|}
\hline $\mathrm{nr}$ & type & resnr & residue & atom & cgnr & charge \\
\hline 1 & SN0 & 1 & PEO & O1 & 1 & 0 \\
\hline 2 & SN0 & 1 & PEO & O1 & 2 & 0 \\
\hline 3 & SN0 & 1 & PEO & O1 & 3 & 0 \\
\hline 4 & SN0 & 1 & PEO & $\mathrm{O} 1$ & 4 & 0 \\
\hline 5 & SN0 & 1 & PEO & O1 & 5 & 0 \\
\hline 6 & SN0 & 1 & PEO & $\mathrm{O} 1$ & 6 & 0 \\
\hline 7 & SN0 & 1 & PEO & O1 & 7 & 0 \\
\hline 8 & SN0 & 1 & PEO & $\mathrm{O} 1$ & 8 & 0 \\
\hline 9 & SN0 & 1 & PEO & O1 & 9 & 0 \\
\hline 10 & SN0 & 1 & PEO & $\mathrm{O} 1$ & 10 & 0 \\
\hline 11 & SN0 & 1 & PEO & $\mathrm{O} 1$ & 11 & 0 \\
\hline 12 & SN0 & 1 & PEO & $\mathrm{O} 1$ & 12 & 0 \\
\hline 13 & SN0 & 1 & PEO & $\mathrm{O} 1$ & 13 & 0 \\
\hline 14 & SN0 & 1 & PEO & O1 & 14 & 0 \\
\hline 15 & SN0 & 1 & PEO & O1 & 15 & 0 \\
\hline 16 & SN0 & 1 & PEO & O1 & 16 & 0 \\
\hline 17 & SN0 & 1 & PEO & O1 & 17 & 0 \\
\hline 18 & SN0 & 1 & PEO & O1 & 18 & 0 \\
\hline 19 & SN0 & 1 & PEO & O1 & 19 & 0 \\
\hline 20 & SN0 & 1 & PEO & O1 & 20 & 0 \\
\hline 21 & SN0 & 1 & PEO & O1 & 21 & 0 \\
\hline 22 & SN0 & 1 & PEO & O1 & 22 & 0 \\
\hline 23 & SN0 & 1 & PEO & O1 & 23 & 0 \\
\hline 24 & SN0 & 1 & PEO & O1 & 24 & 0 \\
\hline 25 & SN0 & 1 & PEO & O1 & 25 & 0 \\
\hline 26 & SN0 & 1 & PEO & O1 & 26 & 0 \\
\hline 27 & SN0 & 1 & PEO & O1 & 27 & 0 \\
\hline 28 & SN0 & 1 & PEO & O1 & 28 & 0 \\
\hline 29 & SN0 & 1 & PEO & O1 & 29 & 0 \\
\hline 30 & SN0 & 1 & PEO & O1 & 30 & 0 \\
\hline 31 & SN0 & 1 & PEO & O1 & 31 & 0 \\
\hline 32 & SN0 & 1 & PEO & O1 & 32 & 0 \\
\hline 33 & SN0 & 1 & PEO & O1 & 33 & 0 \\
\hline 34 & SN0 & 1 & PEO & O1 & 34 & 0 \\
\hline 35 & SN0 & 1 & PEO & O1 & 35 & 0 \\
\hline 36 & SN0 & 1 & PEO & O1 & 36 & 0 \\
\hline 37 & SN0 & 1 & PEO & O1 & 37 & 0 \\
\hline 38 & SNO & 1 & PEO & $\mathrm{O} 1$ & 38 & 0 \\
\hline
\end{tabular}




\begin{tabular}{|c|c|c|c|c|c|c|}
\hline 39 & SN0 & 1 & PEO & $\mathrm{O} 1$ & 39 & 0 \\
\hline 40 & SN0 & 1 & PEO & $\mathrm{O} 1$ & 40 & 0 \\
\hline 41 & SN0 & 1 & PEO & $\mathrm{O} 1$ & 41 & 0 \\
\hline 42 & SN0 & 1 & PEO & $\mathrm{O} 1$ & 42 & 0 \\
\hline 43 & SN0 & 1 & PEO & $\mathrm{O} 1$ & 43 & 0 \\
\hline 44 & SN0 & 1 & PEO & $\mathrm{O} 1$ & 44 & 0 \\
\hline 45 & SN0 & 1 & PEO & $\mathrm{O} 1$ & 45 & 0 \\
\hline 46 & SN0 & 1 & PEO & $\mathrm{O} 1$ & 46 & 0 \\
\hline 47 & SN0 & 1 & PEO & $\mathrm{O} 1$ & 47 & 0 \\
\hline 48 & SN0 & 1 & PEO & $\mathrm{O} 1$ & 48 & 0 \\
\hline 49 & SN0 & 1 & PEO & $\mathrm{O} 1$ & 49 & 0 \\
\hline 50 & SN0 & 1 & PEO & $\mathrm{O} 1$ & 50 & 0 \\
\hline 51 & SN0 & 1 & PEO & $\mathrm{O} 1$ & 51 & 0 \\
\hline 52 & SN0 & 1 & PEO & $\mathrm{O} 1$ & 52 & 0 \\
\hline 53 & SN0 & 1 & PEO & $\mathrm{O} 1$ & 53 & 0 \\
\hline 54 & SN0 & 1 & PEO & $\mathrm{O} 1$ & 54 & 0 \\
\hline 55 & SN0 & 1 & PEO & $\mathrm{O} 1$ & 55 & 0 \\
\hline 56 & SN0 & 1 & PEO & $\mathrm{O} 1$ & 56 & 0 \\
\hline 57 & SN0 & 1 & PEO & $\mathrm{O} 1$ & 57 & 0 \\
\hline 58 & SN0 & 1 & PEO & $\mathrm{O} 1$ & 58 & 0 \\
\hline 59 & SN0 & 1 & PEO & $\mathrm{O} 1$ & 59 & 0 \\
\hline 60 & SN0 & 1 & PEO & $\mathrm{O} 1$ & 60 & 0 \\
\hline 61 & SN0 & 1 & PEO & $\mathrm{O} 1$ & 61 & 0 \\
\hline 62 & SN0 & 1 & PEO & $\mathrm{O} 1$ & 62 & 0 \\
\hline 63 & SN0 & 1 & PEO & $\mathrm{O} 1$ & 63 & 0 \\
\hline 64 & SN0 & 1 & PEO & $\mathrm{O} 1$ & 64 & 0 \\
\hline 65 & SN0 & 1 & PEO & $\mathrm{O} 1$ & 65 & 0 \\
\hline 66 & SN0 & 1 & PEO & $\mathrm{O} 1$ & 66 & 0 \\
\hline 67 & SN0 & 1 & PEO & $\mathrm{O} 1$ & 67 & 0 \\
\hline 68 & SN0 & 1 & PEO & $\mathrm{O} 1$ & 68 & 0 \\
\hline 69 & SN0 & 1 & PEO & $\mathrm{O} 1$ & 69 & 0 \\
\hline 70 & SN0 & 1 & PEO & $\mathrm{O} 1$ & 70 & 0 \\
\hline 71 & SN0 & 1 & PEO & $\mathrm{O} 1$ & 71 & 0 \\
\hline 72 & SN0 & 1 & PEO & $\mathrm{O} 1$ & 72 & 0 \\
\hline 73 & SN0 & 1 & PEO & $\mathrm{O} 1$ & 73 & 0 \\
\hline 74 & SN0 & 1 & PEO & $\mathrm{O} 1$ & 74 & 0 \\
\hline 75 & SN0 & 1 & PEO & $\mathrm{O} 1$ & 75 & 0 \\
\hline 76 & SN0 & 1 & PEO & $\mathrm{O} 1$ & 76 & 0 \\
\hline 77 & SN0 & 1 & PEO & $\mathrm{O} 1$ & 77 & 0 \\
\hline 78 & SN0 & 1 & PEO & $\mathrm{O} 1$ & 78 & 0 \\
\hline 79 & SN0 & 1 & PEO & $\mathrm{O} 1$ & 79 & 0 \\
\hline 80 & SN0 & 1 & PEO & $\mathrm{O} 1$ & 80 & 0 \\
\hline
\end{tabular}




\begin{tabular}{|c|c|c|c|c|c|c|}
\hline 81 & SN0 & 1 & PEO & O1 & 81 & 0 \\
\hline 82 & SN0 & 1 & PEO & O1 & 82 & 0 \\
\hline 83 & SN0 & 1 & PEO & O1 & 83 & 0 \\
\hline 84 & SN0 & 1 & PEO & O1 & 84 & 0 \\
\hline 85 & SN0 & 1 & PEO & O1 & 85 & 0 \\
\hline 86 & SN0 & 1 & PEO & $\mathrm{O} 1$ & 86 & 0 \\
\hline 87 & SN0 & 1 & PEO & O1 & 87 & 0 \\
\hline 88 & SN0 & 1 & PEO & O1 & 88 & 0 \\
\hline 89 & SN0 & 1 & PEO & O1 & 89 & 0 \\
\hline 90 & SN0 & 1 & PEO & $\mathrm{O} 1$ & 90 & 0 \\
\hline 91 & SN0 & 1 & PEO & O1 & 91 & 0 \\
\hline 92 & SN0 & 1 & PEO & O1 & 92 & 0 \\
\hline 93 & SN0 & 1 & PEO & O1 & 93 & 0 \\
\hline 94 & SN0 & 1 & PEO & $\mathrm{O} 1$ & 94 & 0 \\
\hline 95 & SN0 & 1 & PEO & O1 & 95 & 0 \\
\hline 96 & SN0 & 1 & PEO & O1 & 96 & 0 \\
\hline 97 & SN0 & 1 & PEO & $\mathrm{O} 1$ & 97 & 0 \\
\hline 98 & SN0 & 1 & PEO & $\mathrm{O} 1$ & 98 & 0 \\
\hline 99 & SN0 & 1 & PEO & O1 & 99 & 0 \\
\hline 100 & SN0 & 1 & PEO & O1 & 100 & 0 \\
\hline 101 & SN0 & 1 & PEO & O1 & 101 & 0 \\
\hline 102 & SN0 & 1 & PEO & O1 & 102 & 0 \\
\hline 103 & SN0 & 1 & PEO & O1 & 103 & 0 \\
\hline 104 & SN0 & 1 & PEO & O1 & 104 & 0 \\
\hline 105 & SN0 & 1 & PEO & O1 & 105 & 0 \\
\hline 106 & SN0 & 1 & PEO & O1 & 106 & 0 \\
\hline 107 & SN0 & 1 & PEO & O1 & 107 & 0 \\
\hline 108 & SN0 & 1 & PEO & O1 & 108 & 0 \\
\hline 109 & SN0 & 1 & PEO & O1 & 109 & 0 \\
\hline 110 & SN0 & 1 & PEO & O1 & 110 & 0 \\
\hline 111 & SN0 & 1 & PEO & O1 & 111 & 0 \\
\hline 112 & SN0 & 1 & PEO & O1 & 112 & 0 \\
\hline 113 & P5 & 2 & LYS & BB & 113 & 0 \\
\hline 114 & $\mathrm{C} 3$ & 2 & LYS & SC1 & 114 & 0 \\
\hline 115 & $\mathrm{P} 1$ & 2 & LYS & $\mathrm{SC} 2$ & 115 & 0 \\
\hline 116 & P5 & 3 & LYS & BB & 116 & 0 \\
\hline 117 & $\mathrm{C} 3$ & 3 & LYS & SC1 & 117 & 0 \\
\hline 118 & $\mathrm{P} 1$ & 3 & LYS & $\mathrm{SC} 2$ & 118 & 0 \\
\hline 119 & P5 & 4 & LYS & $\mathrm{BB}$ & 119 & 0 \\
\hline 120 & $\mathrm{C} 3$ & 4 & LYS & SC1 & 120 & 0 \\
\hline 121 & P1 & 4 & LYS & SC2 & 121 & 0 \\
\hline 122 & P5 & 5 & LYS & $\mathrm{BB}$ & 122 & 0 \\
\hline
\end{tabular}




\begin{tabular}{|c|c|c|c|c|c|c|}
\hline 123 & C3 & 5 & LYS & $\mathrm{SC} 1$ & 123 & 0 \\
\hline 124 & P1 & 5 & LYS & $\mathrm{SC} 2$ & 124 & 0 \\
\hline 125 & SP5 & 6 & CHOA & $\mathrm{OH}$ & 125 & 0 \\
\hline 126 & $\mathrm{SC} 1$ & 6 & CHOA & R1 & 126 & 0 \\
\hline 127 & $\mathrm{SC} 3$ & 6 & CHOA & R2 & 127 & 0 \\
\hline 128 & SP5 & 6 & CHOA & $\mathrm{O} 2$ & 128 & 0 \\
\hline 129 & SP5 & 6 & CHOA & $\mathrm{O} 3$ & 129 & 0 \\
\hline 130 & $\mathrm{SC} 1$ & 6 & CHOA & R3 & 130 & 0 \\
\hline 131 & $\mathrm{SC} 1$ & 6 & CHOA & $\mathrm{C} 1$ & 131 & 0 \\
\hline 132 & SP5 & 7 & CHOA & $\mathrm{OH}$ & 132 & 0 \\
\hline 133 & $\mathrm{SC} 1$ & 7 & CHOA & R1 & 133 & 0 \\
\hline 134 & $\mathrm{SC} 3$ & 7 & CHOA & R2 & 134 & 0 \\
\hline 135 & SP5 & 7 & CHOA & $\mathrm{O} 2$ & 135 & 0 \\
\hline 136 & SP5 & 7 & CHOA & $\mathrm{O} 3$ & 136 & 0 \\
\hline 137 & $\mathrm{SC} 1$ & 7 & CHOA & R3 & 137 & 0 \\
\hline 138 & $\mathrm{SC} 1$ & 7 & CHOA & $\mathrm{C} 1$ & 138 & 0 \\
\hline 139 & SP5 & 8 & CHOA & $\mathrm{OH}$ & 139 & 0 \\
\hline 140 & $\mathrm{SC} 1$ & 8 & CHOA & R1 & 140 & 0 \\
\hline 141 & $\mathrm{SC} 3$ & 8 & CHOA & R2 & 141 & 0 \\
\hline 142 & SP5 & 8 & CHOA & $\mathrm{O} 2$ & 142 & 0 \\
\hline 143 & SP5 & 8 & CHOA & $\mathrm{O} 3$ & 143 & 0 \\
\hline 144 & $\mathrm{SC} 1$ & 8 & CHOA & R3 & 144 & 0 \\
\hline 145 & $\mathrm{SC} 1$ & 8 & CHOA & $\mathrm{C} 1$ & 145 & 0 \\
\hline 146 & SP5 & 9 & CHOA & $\mathrm{OH}$ & 146 & 0 \\
\hline 147 & $\mathrm{SC} 1$ & 9 & CHOA & R1 & 147 & 0 \\
\hline 148 & $\mathrm{SC} 3$ & 9 & CHOA & R2 & 148 & 0 \\
\hline 149 & SP5 & 9 & CHOA & $\mathrm{O} 2$ & 149 & 0 \\
\hline 150 & SP5 & 9 & CHOA & $\mathrm{O} 3$ & 150 & 0 \\
\hline 151 & $\mathrm{SC} 1$ & 9 & CHOA & R3 & 151 & 0 \\
\hline 152 & $\mathrm{SC} 1$ & 9 & CHOA & $\mathrm{C} 1$ & 152 & 0 \\
\hline 153 & SNO & 10 & LIN & O1 & 153 & 0 \\
\hline 154 & SNO & 10 & LIN & $\mathrm{O} 1$ & 154 & 0 \\
\hline 155 & SNO & 10 & LIN & O1 & 155 & 0 \\
\hline 156 & SN0 & 10 & LIN & O1 & 156 & 0 \\
\hline 157 & SN0 & 10 & LIN & O1 & 157 & 0 \\
\hline 158 & P5 & 11 & LYS & BB & 158 & 0 \\
\hline 159 & C3 & 11 & LYS & $\mathrm{SC} 1$ & 159 & 0 \\
\hline 160 & P1 & 11 & LYS & $\mathrm{SC} 2$ & 160 & 0 \\
\hline 161 & P5 & 12 & LYS & BB & 161 & 0 \\
\hline 162 & $\mathrm{C} 3$ & 12 & LYS & $\mathrm{SC} 1$ & 162 & 0 \\
\hline 163 & P1 & 12 & LYS & $\mathrm{SC} 2$ & 163 & 0 \\
\hline 164 & P5 & 13 & LYS & BB & 164 & 0 \\
\hline
\end{tabular}




\begin{tabular}{lllllll}
\hline 165 & C3 & 13 & LYS & SC1 & 165 & 0 \\
166 & P1 & 13 & LYS & SC2 & 166 & 0 \\
167 & SP5 & 14 & RHN & N1 & 167 & 0 \\
168 & SC4 & 14 & RHN & N2 & 168 & 0 \\
169 & SC1 & 14 & RHN & N3 & 169 & 0 \\
170 & P2 & 14 & RHN & N4 & 170 & 0 \\
171 & P2 & 14 & RHN & N5 & 171 & 0 \\
172 & SC4 & 14 & RHN & N6 & 172 & 0 \\
173 & SC1 & 14 & RHN & N7 & 173 & 0 \\
174 & SP5 & 14 & RHN & N8 & 174 & 0 \\
175 & P3 & 14 & RHN & N9 & 175 & 0 \\
176 & SP5 & 15 & RHN & N1 & 176 & 0 \\
177 & SC4 & 15 & RHN & N2 & 177 & 0 \\
178 & SC1 & 15 & RHN & N3 & 178 & 0 \\
179 & P2 & 15 & RHN & N4 & 179 & 0 \\
180 & P2 & 15 & RHN & N5 & 180 & 0 \\
181 & SC4 & 15 & RHN & N6 & 181 & 0 \\
182 & SC1 & 15 & RHN & N7 & 182 & 0 \\
183 & SP5 & 15 & RHN & N8 & 183 & 0 \\
184 & P3 & 15 & RHN & N9 & 184 & 0 \\
185 & SP5 & 16 & RHN & N1 & 185 & 0 \\
186 & SC4 & 16 & RHN & N2 & 186 & 0 \\
187 & SC1 & 16 & RHN & N3 & 187 & 0 \\
188 & P2 & 16 & RHN & N4 & 188 & 0 \\
189 & P2 & 16 & RHN & N5 & 189 & 0 \\
190 & SC4 & 16 & RHN & N6 & 190 & 0 \\
191 & SC1 & 16 & RHN & N7 & 191 & 0 \\
192 & SP5 & 16 & RHN & N8 & 192 & 0 \\
193 & P3 & 16 & RHN & N9 & 193 & 0 \\
194 & SP5 & 17 & RHN & N1 & 194 & 0 \\
195 & SC4 & 17 & RHN & N2 & 195 & 0 \\
196 & SC1 & 17 & RHN & N3 & 196 & 0 \\
197 & P2 & 17 & RHN & N4 & 197 & 0 \\
198 & P2 & 17 & RHN & N5 & 198 & 0 \\
199 & SC4 & 17 & RHN & N6 & 199 & 0 \\
200 & SC1 17 & RHN & N7 & 200 & 0 \\
201 & SP5 & 17 & NHN & N9 & 201 & 0 \\
202 & P3 & 1702 & 0 \\
\hline & & & & & \\
\hline
\end{tabular}


Table S6. Bond length parameters for coarse-grained $\mathrm{PEG}^{5 \mathrm{~K}} \mathrm{CA}_{4}-\mathrm{L}-\mathrm{RH}_{4}$ telodendrimer.

\begin{tabular}{rrrrr}
\hline $\mathrm{i}$ & $\mathrm{j}$ & funct & length & force.c. \\
\hline 1 & 2 & 1 & 0.33 & 17000 \\
2 & 3 & 1 & 0.33 & 17000 \\
3 & 4 & 1 & 0.33 & 17000 \\
4 & 5 & 1 & 0.33 & 17000 \\
5 & 6 & 1 & 0.33 & 17000 \\
6 & 7 & 1 & 0.33 & 17000 \\
7 & 8 & 1 & 0.33 & 17000 \\
8 & 9 & 1 & 0.33 & 17000 \\
9 & 10 & 1 & 0.33 & 17000 \\
10 & 11 & 1 & 0.33 & 17000 \\
11 & 12 & 1 & 0.33 & 17000 \\
12 & 13 & 1 & 0.33 & 17000 \\
13 & 14 & 1 & 0.33 & 17000 \\
14 & 15 & 1 & 0.33 & 17000 \\
15 & 16 & 1 & 0.33 & 17000 \\
16 & 17 & 1 & 0.33 & 17000 \\
17 & 18 & 1 & 0.33 & 17000 \\
18 & 19 & 1 & 0.33 & 17000 \\
19 & 20 & 1 & 0.33 & 17000 \\
20 & 21 & 1 & 0.33 & 17000 \\
21 & 22 & 1 & 0.33 & 17000 \\
22 & 23 & 1 & 0.33 & 17000 \\
23 & 24 & 1 & 0.33 & 17000 \\
24 & 25 & 1 & 0.33 & 17000 \\
25 & 26 & 1 & 0.33 & 17000 \\
26 & 27 & 1 & 0.33 & 17000 \\
27 & 28 & 1 & 0.33 & 17000 \\
28 & 29 & 1 & 0.33 & 17000 \\
29 & 30 & 1 & 0.33 & 17000 \\
30 & 31 & 1 & 0.33 & 17000 \\
31 & 32 & 1 & 0.33 & 17000 \\
32 & 33 & 1 & 0.33 & 17000 \\
33 & 34 & 1 & 0.33 & 17000 \\
34 & 35 & 1 & 0.33 & 17000 \\
35 & 36 & 1 & 0.33 & 17000 \\
36 & 37 & 1 & 0.33 & 17000 \\
37 & 38 & 1 & 0.33 & 17000 \\
38 & 39 & 1 & 0.33 & 17000 \\
39 & 40 & 1 & 0.33 & 17000 \\
40 & 41 & 1 & 0.33 & 17000 \\
& & & &
\end{tabular}




$\begin{array}{lllll}41 & 42 & 1 & 0.33 & 17000 \\ 42 & 43 & 1 & 0.33 & 17000 \\ 43 & 44 & 1 & 0.33 & 17000 \\ 44 & 45 & 1 & 0.33 & 17000 \\ 45 & 46 & 1 & 0.33 & 17000 \\ 46 & 47 & 1 & 0.33 & 17000 \\ 47 & 48 & 1 & 0.33 & 17000 \\ 48 & 49 & 1 & 0.33 & 17000 \\ 49 & 50 & 1 & 0.33 & 17000 \\ 50 & 51 & 1 & 0.33 & 17000 \\ 51 & 52 & 1 & 0.33 & 17000 \\ 52 & 53 & 1 & 0.33 & 17000 \\ 53 & 54 & 1 & 0.33 & 17000 \\ 54 & 55 & 1 & 0.33 & 17000 \\ 55 & 56 & 1 & 0.33 & 17000 \\ 56 & 57 & 1 & 0.33 & 17000 \\ 57 & 58 & 1 & 0.33 & 17000 \\ 58 & 59 & 1 & 0.33 & 17000 \\ 59 & 60 & 1 & 0.33 & 17000 \\ 60 & 61 & 1 & 0.33 & 17000 \\ 61 & 62 & 1 & 0.33 & 17000 \\ 62 & 63 & 1 & 0.33 & 17000 \\ 63 & 64 & 1 & 0.33 & 17000 \\ 64 & 65 & 1 & 0.33 & 17000 \\ 65 & 66 & 1 & 0.33 & 17000 \\ 66 & 67 & 1 & 0.33 & 17000 \\ 67 & 68 & 1 & 0.33 & 17000 \\ 68 & 69 & 1 & 0.33 & 17000 \\ 69 & 70 & 1 & 0.33 & 17000 \\ 70 & 71 & 1 & 0.33 & 17000 \\ 71 & 72 & 1 & 0.33 & 17000 \\ 72 & 73 & 1 & 0.33 & 17000 \\ 73 & 74 & 1 & 0.33 & 17000 \\ 74 & 75 & 1 & 0.33 & 17000 \\ 75 & 76 & 1 & 0.33 & 17000 \\ 76 & 77 & 1 & 0.33 & 17000 \\ 77 & 78 & 1 & 0.33 & 17000 \\ 78 & 79 & 1 & 0.33 & 17000 \\ 79 & 80 & 1 & 0.33 & 17000 \\ 80 & 81 & 0.33 & 17000 \\ 81 & 82 & 0.33 & 17000 \\ & 83 & 0.33 & 17000\end{array}$




$\begin{array}{rrrrr}83 & 84 & 1 & 0.33 & 17000 \\ 84 & 85 & 1 & 0.33 & 17000 \\ 85 & 86 & 1 & 0.33 & 17000 \\ 86 & 87 & 1 & 0.33 & 17000 \\ 87 & 88 & 1 & 0.33 & 17000 \\ 88 & 89 & 1 & 0.33 & 17000 \\ 89 & 90 & 1 & 0.33 & 17000 \\ 90 & 91 & 1 & 0.33 & 17000 \\ 91 & 92 & 1 & 0.33 & 17000 \\ 92 & 93 & 1 & 0.33 & 17000 \\ 93 & 94 & 1 & 0.33 & 17000 \\ 94 & 95 & 1 & 0.33 & 17000 \\ 95 & 96 & 1 & 0.33 & 17000 \\ 96 & 97 & 1 & 0.33 & 17000 \\ 97 & 98 & 1 & 0.33 & 17000 \\ 98 & 99 & 1 & 0.33 & 17000 \\ 99 & 100 & 1 & 0.33 & 17000 \\ 100 & 101 & 1 & 0.33 & 17000 \\ 101 & 102 & 1 & 0.33 & 17000 \\ 102 & 103 & 1 & 0.33 & 17000 \\ 103 & 104 & 1 & 0.33 & 17000 \\ 104 & 105 & 1 & 0.33 & 17000 \\ 105 & 106 & 1 & 0.33 & 17000 \\ 106 & 107 & 1 & 0.33 & 17000 \\ 107 & 108 & 1 & 0.33 & 17000 \\ 108 & 109 & 1 & 0.33 & 17000 \\ 109 & 110 & 1 & 0.33 & 17000 \\ 110 & 111 & 1 & 0.33 & 17000 \\ 111 & 112 & 1 & 0.33 & 17000 \\ 112 & 119 & 1 & 0.33 & 1200 \\ 113 & 116 & 1 & 0.35 & 1250 \\ 116 & 119 & 1 & 0.35 & 1250 \\ 121 & 122 & 1 & 0.35 & 1250 \\ 113 & 114 & 1 & 0.33 & 5000 \\ 116 & 117 & 1 & 0.33 & 5000 \\ 119 & 120 & 1 & 0.33 & 5000 \\ 122 & 123 & 1 & 0.33 & 5000 \\ 114 & 115 & 1 & 0.28 & 5000 \\ 117 & 118 & 1 & 0.28 & 5000 \\ 120 & 121 & 1 & 0.28 & 5000 \\ 123 & 124 & 1 & 0.28 & 5000 \\ 115 & 131 & 1 & 0.368 & 20000\end{array}$




$\begin{array}{rrrrr}113 & 138 & 1 & 0.368 & 20000 \\ 122 & 145 & 1 & 0.368 & 20000 \\ 124 & 152 & 1 & 0.368 & 20000 \\ 125 & 126 & 1 & 0.242 & 20000 \\ 126 & 127 & 1 & 0.26 & 20000 \\ 126 & 128 & 1 & 0.341 & 20000 \\ 128 & 130 & 1 & 0.213 & 20000 \\ 128 & 131 & 1 & 0.544 & 20000 \\ 129 & 130 & 1 & 0.203 & 20000 \\ 130 & 131 & 1 & 0.368 & 20000 \\ 132 & 133 & 1 & 0.242 & 20000 \\ 133 & 134 & 1 & 0.26 & 20000 \\ 133 & 135 & 1 & 0.341 & 20000 \\ 135 & 137 & 1 & 0.213 & 20000 \\ 135 & 138 & 1 & 0.544 & 20000 \\ 136 & 137 & 1 & 0.203 & 20000 \\ 137 & 138 & 1 & 0.368 & 20000 \\ 139 & 140 & 1 & 0.242 & 20000 \\ 140 & 141 & 1 & 0.26 & 20000 \\ 140 & 142 & 1 & 0.341 & 20000 \\ 142 & 144 & 1 & 0.213 & 20000 \\ 142 & 145 & 1 & 0.544 & 20000 \\ 143 & 144 & 1 & 0.203 & 20000 \\ 144 & 145 & 1 & 0.368 & 20000 \\ 146 & 147 & 1 & 0.242 & 20000 \\ 147 & 148 & 1 & 0.26 & 20000 \\ 147 & 149 & 1 & 0.341 & 20000 \\ 149 & 151 & 1 & 0.213 & 20000 \\ 149 & 152 & 1 & 0.544 & 20000 \\ 150 & 151 & 1 & 0.203 & 20000 \\ 151 & 152 & 1 & 0.368 & 20000 \\ 153 & 118 & 1 & 0.33 & 1200 \\ 153 & 154 & 1 & 0.33 & 17000 \\ 154 & 155 & 1 & 0.33 & 17000 \\ 155 & 156 & 1 & 0.33 & 17000 \\ 156 & 157 & 1 & 0.33 & 17000 \\ 157 & 161 & 1 & 0.33 & 1200 \\ 158 & 161 & 1 & 0.35 & 1250 \\ 163 & 164 & 1 & 0.35 & 1250 \\ 158 & 159 & 1 & 0.33 & 5000 \\ 161 & 162 & 1 & 0.33 & 5000 \\ 164 & 165 & 1 & 0.33 & 5000\end{array}$




$\begin{array}{rrrrr}159 & 160 & 1 & 0.28 & 5000 \\ 162 & 163 & 1 & 0.28 & 5000 \\ 165 & 166 & 1 & 0.28 & 5000 \\ 160 & 175 & 1 & 0.368 & 20000 \\ 158 & 184 & 1 & 0.368 & 20000 \\ 164 & 193 & 1 & 0.368 & 20000 \\ 166 & 202 & 1 & 0.368 & 20000 \\ 167 & 168 & 1 & 0.27 & 9000 \\ 168 & 169 & 1 & 0.27 & 9000 \\ 169 & 167 & 1 & 0.27 & 9000 \\ 172 & 173 & 1 & 0.27 & 9000 \\ 173 & 174 & 1 & 0.27 & 9000 \\ 174 & 172 & 1 & 0.27 & 9000 \\ 168 & 170 & 1 & 0.27 & 9000 \\ 168 & 171 & 1 & 0.27 & 9000 \\ 170 & 172 & 1 & 0.27 & 9000 \\ 171 & 172 & 1 & 0.27 & 9000 \\ 170 & 171 & 1 & 0.415 & 9000 \\ 173 & 175 & 1 & 0.231 & 9000 \\ 176 & 177 & 1 & 0.27 & 9000 \\ 177 & 178 & 1 & 0.27 & 9000 \\ 178 & 176 & 1 & 0.27 & 9000 \\ 181 & 182 & 1 & 0.27 & 9000 \\ 182 & 183 & 1 & 0.27 & 9000 \\ 183 & 181 & 1 & 0.27 & 9000 \\ 177 & 179 & 1 & 0.27 & 9000 \\ 177 & 180 & 1 & 0.27 & 9000 \\ 179 & 181 & 1 & 0.27 & 9000 \\ 180 & 181 & 1 & 0.27 & 9000 \\ 179 & 180 & 1 & 0.415 & 9000 \\ 182 & 184 & 1 & 0.231 & 9000 \\ 185 & 186 & 1 & 0.27 & 9000 \\ 186 & 187 & 1 & 0.27 & 9000 \\ 187 & 185 & 1 & 0.27 & 9000 \\ 190 & 191 & 1 & 0.27 & 9000 \\ 191 & 192 & 1 & 0.27 & 9000 \\ 192 & 190 & 1 & 0.27 & 9000 \\ 186 & 188 & 1 & 0.27 & 9000 \\ 186 & 189 & 1 & 0.27 & 9000 \\ 188 & 190 & 1 & 0.27 & 9000 \\ 189 & 190 & 1 & 0.27 & 9000 \\ 188 & 189 & 1 & 0.415 & 9000\end{array}$




\begin{tabular}{rrrrr}
191 & 193 & 1 & 0.231 & 9000 \\
194 & 195 & 1 & 0.27 & 9000 \\
195 & 196 & 1 & 0.27 & 9000 \\
196 & 194 & 1 & 0.27 & 9000 \\
199 & 200 & 1 & 0.27 & 9000 \\
200 & 201 & 1 & 0.27 & 9000 \\
201 & 199 & 1 & 0.27 & 9000 \\
195 & 197 & 1 & 0.27 & 9000 \\
195 & 198 & 1 & 0.27 & 9000 \\
197 & 199 & 1 & 0.27 & 9000 \\
198 & 199 & 1 & 0.27 & 9000 \\
197 & 198 & 1 & 0.415 & 9000 \\
200 & 202 & 1 & 0.231 & 9000 \\
\hline
\end{tabular}


Table S7. Angle parameters for coarse-grained $\mathrm{PEG}^{5 \mathrm{~K}} \mathrm{CA}_{4}-\mathrm{L}-\mathrm{RH}_{4}$ telodendrimer.

\begin{tabular}{|c|c|c|c|c|c|}
\hline $\mathrm{i}$ & $\mathrm{j}$ & $\mathrm{k}$ & funct & angle & force.c. \\
\hline 1 & 2 & 3 & 2 & 130 & 50 \\
\hline 2 & 3 & 4 & 2 & 130 & 50 \\
\hline 3 & 4 & 5 & 2 & 130 & 50 \\
\hline 4 & 5 & 6 & 2 & 130 & 50 \\
\hline 5 & 6 & 7 & 2 & 130 & 50 \\
\hline 6 & 7 & 8 & 2 & 130 & 50 \\
\hline 7 & 8 & 9 & 2 & 130 & 50 \\
\hline 8 & 9 & 10 & 2 & 130 & 50 \\
\hline 9 & 10 & 11 & 2 & 130 & 50 \\
\hline 10 & 11 & 12 & 2 & 130 & 50 \\
\hline 11 & 12 & 13 & 2 & 130 & 50 \\
\hline 12 & 13 & 14 & 2 & 130 & 50 \\
\hline 13 & 14 & 15 & 2 & 130 & 50 \\
\hline 14 & 15 & 16 & 2 & 130 & 50 \\
\hline 15 & 16 & 17 & 2 & 130 & 50 \\
\hline 16 & 17 & 18 & 2 & 130 & 50 \\
\hline 17 & 18 & 19 & 2 & 130 & 50 \\
\hline 18 & 19 & 20 & 2 & 130 & 50 \\
\hline 19 & 20 & 21 & 2 & 130 & 50 \\
\hline 20 & 21 & 22 & 2 & 130 & 50 \\
\hline 21 & 22 & 23 & 2 & 130 & 50 \\
\hline 22 & 23 & 24 & 2 & 130 & 50 \\
\hline 23 & 24 & 25 & 2 & 130 & 50 \\
\hline 24 & 25 & 26 & 2 & 130 & 50 \\
\hline 25 & 26 & 27 & 2 & 130 & 50 \\
\hline 26 & 27 & 28 & 2 & 130 & 50 \\
\hline 27 & 28 & 29 & 2 & 130 & 50 \\
\hline 28 & 29 & 30 & 2 & 130 & 50 \\
\hline 29 & 30 & 31 & 2 & 130 & 50 \\
\hline 30 & 31 & 32 & 2 & 130 & 50 \\
\hline 31 & 32 & 33 & 2 & 130 & 50 \\
\hline 32 & 33 & 34 & 2 & 130 & 50 \\
\hline 33 & 34 & 35 & 2 & 130 & 50 \\
\hline 34 & 35 & 36 & 2 & 130 & 50 \\
\hline 35 & 36 & 37 & 2 & 130 & 50 \\
\hline 36 & 37 & 38 & 2 & 130 & 50 \\
\hline 37 & 38 & 39 & 2 & 130 & 50 \\
\hline 38 & 39 & 40 & 2 & 130 & 50 \\
\hline 39 & 40 & 41 & 2 & 130 & 50 \\
\hline 40 & 41 & 42 & 2 & 130 & 50 \\
\hline
\end{tabular}




$\begin{array}{llllll}41 & 42 & 43 & 2 & 130 & 50 \\ 42 & 43 & 44 & 2 & 130 & 50 \\ 43 & 44 & 45 & 2 & 130 & 50 \\ 44 & 45 & 46 & 2 & 130 & 50 \\ 45 & 46 & 47 & 2 & 130 & 50 \\ 46 & 47 & 48 & 2 & 130 & 50 \\ 47 & 48 & 49 & 2 & 130 & 50 \\ 48 & 49 & 50 & 2 & 130 & 50 \\ 49 & 50 & 51 & 2 & 130 & 50 \\ 50 & 51 & 52 & 2 & 130 & 50 \\ 51 & 52 & 53 & 2 & 130 & 50 \\ 52 & 53 & 54 & 2 & 130 & 50 \\ 53 & 54 & 55 & 2 & 130 & 50 \\ 54 & 55 & 56 & 2 & 130 & 50 \\ 55 & 56 & 57 & 2 & 130 & 50 \\ 56 & 57 & 58 & 2 & 130 & 50 \\ 57 & 58 & 59 & 2 & 130 & 50 \\ 58 & 59 & 60 & 2 & 130 & 50 \\ 59 & 60 & 61 & 2 & 130 & 50 \\ 60 & 61 & 62 & 2 & 130 & 50 \\ 61 & 62 & 63 & 2 & 130 & 50 \\ 62 & 63 & 64 & 2 & 130 & 50 \\ 63 & 64 & 65 & 2 & 130 & 50 \\ 64 & 65 & 66 & 2 & 130 & 50 \\ 65 & 66 & 67 & 2 & 130 & 50 \\ 66 & 67 & 68 & 2 & 130 & 50 \\ 67 & 68 & 69 & 2 & 130 & 50 \\ 68 & 69 & 70 & 2 & 130 & 50 \\ 69 & 70 & 71 & 2 & 130 & 50 \\ 70 & 71 & 72 & 2 & 130 & 50 \\ 71 & 72 & 73 & 2 & 130 & 50 \\ 72 & 73 & 74 & 2 & 130 & 50 \\ 73 & 74 & 75 & 2 & 130 & 50 \\ 74 & 75 & 76 & 2 & 130 & 50 \\ 75 & 76 & 77 & 2 & 130 & 50 \\ 76 & 77 & 78 & 2 & 130 & 50 \\ 77 & 78 & 79 & 2 & 130 & 50 \\ 78 & 79 & 80 & 81 & 130 & 50 \\ 79 & 80 & 82 & 130 & 50 \\ 80 & 81 & 82 & 53 & 50 \\ 81 & 83 & 84 & 50 & 50 \\ & 53 & & 2 & 50\end{array}$




$\begin{array}{llllll}83 & 84 & 85 & 2 & 130 & 50 \\ 84 & 85 & 86 & 2 & 130 & 50 \\ 85 & 86 & 87 & 2 & 130 & 50 \\ 86 & 87 & 88 & 2 & 130 & 50 \\ 87 & 88 & 89 & 2 & 130 & 50 \\ 88 & 89 & 90 & 2 & 130 & 50 \\ 89 & 90 & 91 & 2 & 130 & 50 \\ 90 & 91 & 92 & 2 & 130 & 50 \\ 91 & 92 & 93 & 2 & 130 & 50 \\ 92 & 93 & 94 & 2 & 130 & 50 \\ 93 & 94 & 95 & 2 & 130 & 50 \\ 94 & 95 & 96 & 2 & 130 & 50 \\ 95 & 96 & 97 & 2 & 130 & 50 \\ 96 & 97 & 98 & 2 & 130 & 50 \\ 97 & 98 & 99 & 2 & 130 & 50 \\ 98 & 99 & 100 & 2 & 130 & 50 \\ 99 & 100 & 101 & 2 & 130 & 50 \\ 100 & 101 & 102 & 2 & 130 & 50 \\ 101 & 102 & 103 & 2 & 130 & 50 \\ 102 & 103 & 104 & 2 & 130 & 50 \\ 103 & 104 & 105 & 2 & 130 & 50 \\ 104 & 105 & 106 & 2 & 130 & 50 \\ 105 & 106 & 107 & 2 & 130 & 50 \\ 106 & 107 & 108 & 2 & 130 & 50 \\ 107 & 108 & 109 & 2 & 130 & 50 \\ 108 & 109 & 110 & 2 & 130 & 50 \\ 109 & 110 & 111 & 2 & 130 & 50 \\ 110 & 111 & 112 & 2 & 130 & 50 \\ 111 & 112 & 119 & 2 & 130 & 50 \\ 112 & 119 & 120 & 2 & 130 & 50 \\ 113 & 116 & 119 & 2 & 134 & 25 \\ 121 & 122 & 123 & 2 & 100 & 25 \\ 116 & 113 & 114 & 2 & 100 & 25 \\ 113 & 116 & 117 & 2 & 100 & 25 \\ 116 & 119 & 120 & 2 & 100 & 25 \\ 113 & 114 & 115 & 2 & 180 & 25 \\ 116 & 117 & 118 & 2 & 180 & 25 \\ 119 & 120 & 121 & 2 & 180 & 25 \\ 122 & 123 & 124 & 2 & 180 & 25 \\ 115 & 131 & 130 & 2 & 130 & 50 \\ 113 & 138 & 137 & 130 & 50 \\ 124 & 152 & 151 & 2 & 130 & 50\end{array}$




$\begin{array}{lccccc}122 & 145 & 144 & 2 & 130 & 50 \\ 154 & 153 & 118 & 2 & 130 & 50 \\ 153 & 118 & 117 & 2 & 130 & 50 \\ 153 & 154 & 155 & 2 & 130 & 50 \\ 154 & 155 & 156 & 2 & 130 & 50 \\ 155 & 156 & 157 & 2 & 130 & 50 \\ 156 & 157 & 161 & 2 & 130 & 50 \\ 157 & 161 & 162 & 2 & 130 & 50 \\ 163 & 164 & 165 & 2 & 100 & 25 \\ 158 & 161 & 162 & 2 & 100 & 25 \\ 158 & 159 & 160 & 2 & 180 & 25 \\ 161 & 162 & 163 & 2 & 180 & 25 \\ 164 & 165 & 166 & 2 & 180 & 25 \\ 160 & 175 & 173 & 2 & 130 & 50 \\ 158 & 184 & 182 & 2 & 130 & 50 \\ 166 & 202 & 200 & 2 & 130 & 50 \\ 164 & 193 & 191 & 2 & 130 & 50 \\ 167 & 168 & 170 & 1 & 78 & 80 \\ 167 & 168 & 171 & 1 & 165 & 80 \\ 167 & 168 & 169 & 1 & 76 & 80 \\ 169 & 168 & 170 & 1 & 178 & 80 \\ 169 & 168 & 171 & 1 & 89 & 80 \\ 174 & 172 & 170 & 1 & 76 & 80 \\ 174 & 172 & 171 & 1 & 165 & 80 \\ 174 & 172 & 173 & 1 & 75 & 80 \\ 173 & 172 & 170 & 1 & 178 & 80 \\ 173 & 172 & 171 & 1 & 91 & 80 \\ 172 & 173 & 175 & 1 & 178 & 80 \\ 168 & 167 & 169 & 1 & 51 & 80 \\ 167 & 169 & 168 & 1 & 56 & 80 \\ 174 & 173 & 172 & 1 & 55 & 80 \\ 172 & 174 & 173 & 1 & 48 & 80 \\ 168 & 170 & 172 & 1 & 60 & 80 \\ 168 & 171 & 172 & 1 & 60 & 80 \\ 176 & 177 & 179 & 1 & 78 & 80 \\ 176 & 177 & 180 & 1 & 165 & 80 \\ 176 & 177 & 178 & 1 & 76 & 80 \\ 178 & 177 & 179 & 1 & 178 & 80 \\ 178 & 177 & 180 & 1 & 89 & 80 \\ 183 & 181 & 179 & 1 & 76 & 80 \\ 183 & 181 & 180 & 185 & 80 \\ 183 & 181 & 182 & 1 & 80\end{array}$




$\begin{array}{lccccc}182 & 181 & 179 & 1 & 178 & 80 \\ 182 & 181 & 180 & 1 & 91 & 80 \\ 181 & 182 & 184 & 1 & 178 & 80 \\ 177 & 176 & 178 & 1 & 51 & 80 \\ 176 & 178 & 177 & 1 & 56 & 80 \\ 183 & 182 & 181 & 1 & 55 & 80 \\ 181 & 183 & 182 & 1 & 48 & 80 \\ 177 & 179 & 181 & 1 & 60 & 80 \\ 177 & 180 & 181 & 1 & 60 & 80 \\ 185 & 186 & 188 & 1 & 78 & 80 \\ 185 & 186 & 189 & 1 & 165 & 80 \\ 185 & 186 & 187 & 1 & 76 & 80 \\ 187 & 186 & 188 & 1 & 178 & 80 \\ 187 & 186 & 189 & 1 & 89 & 80 \\ 192 & 190 & 188 & 1 & 76 & 80 \\ 192 & 190 & 189 & 1 & 165 & 80 \\ 192 & 190 & 191 & 1 & 75 & 80 \\ 191 & 190 & 188 & 1 & 178 & 80 \\ 191 & 190 & 189 & 1 & 91 & 80 \\ 190 & 191 & 193 & 1 & 178 & 80 \\ 186 & 185 & 187 & 1 & 51 & 80 \\ 185 & 187 & 186 & 1 & 56 & 80 \\ 192 & 191 & 190 & 1 & 55 & 80 \\ 190 & 192 & 191 & 1 & 48 & 80 \\ 186 & 188 & 190 & 1 & 60 & 80 \\ 186 & 189 & 190 & 1 & 60 & 80 \\ 194 & 195 & 197 & 1 & 78 & 80 \\ 194 & 195 & 198 & 1 & 165 & 80 \\ 194 & 195 & 196 & 1 & 76 & 80 \\ 196 & 195 & 197 & 1 & 178 & 80 \\ 196 & 195 & 198 & 1 & 89 & 80 \\ 201 & 199 & 197 & 1 & 76 & 80 \\ 201 & 199 & 198 & 1 & 165 & 80 \\ 201 & 199 & 200 & 1 & 75 & 80 \\ 200 & 199 & 197 & 1 & 178 & 80 \\ 200 & 199 & 198 & 1 & 91 & 80 \\ 199 & 200 & 202 & 1 & 178 & 80 \\ 195 & 194 & 196 & 1 & 51 & 80 \\ 194 & 196 & 195 & 1 & 56 & 80 \\ 201 & 200 & 199 & 1 & 55 & 80 \\ 199 & 201 & 200 & 1 & 48 & 80 \\ 195 & 197 & 199 & & & 80\end{array}$




\begin{tabular}{llllll}
195 & 198 & 199 & 1 & 60 & 80 \\
\hline
\end{tabular}

Table S8. Constraints for coarse-grained $\mathrm{PEG}^{5 \mathrm{~K}} \mathrm{CA}_{4}-\mathrm{L}-\mathrm{RH}_{4}$ telodendrimer.

\begin{tabular}{ccrc}
\hline $\mathrm{i}$ & $\mathrm{j}$ & funct & length \\
\hline 125 & 127 & 1 & 0.493 \\
125 & 128 & 1 & 0.604 \\
127 & 128 & 1 & 0.272 \\
127 & 129 & 1 & 0.346 \\
128 & 129 & 1 & 0.294 \\
129 & 131 & 1 & 0.406 \\
132 & 134 & 1 & 0.493 \\
132 & 135 & 1 & 0.604 \\
134 & 135 & 1 & 0.272 \\
134 & 136 & 1 & 0.346 \\
135 & 136 & 1 & 0.294 \\
136 & 138 & 1 & 0.406 \\
139 & 141 & 1 & 0.493 \\
139 & 142 & 1 & 0.604 \\
141 & 142 & 1 & 0.272 \\
141 & 143 & 1 & 0.346 \\
142 & 143 & 1 & 0.294 \\
143 & 145 & 1 & 0.406 \\
146 & 148 & 1 & 0.493 \\
146 & 149 & 1 & 0.604 \\
148 & 149 & 1 & 0.272 \\
148 & 150 & 1 & 0.346 \\
149 & 150 & 1 & 0.294 \\
150 & 152 & 1 & 0.406 \\
\hline
\end{tabular}

